\title{
Etude des agents des myiases des animaux domestiques et sauvages d'Afrique équatoriale
}

\author{
por M. GRABER ef J. GRUVEL \\ Laboratoire de Farcha-Fort-Lamy Tchad
}

\begin{abstract}
RÉSUMÉ
10 Les auteurs notent l'existence des espèces suivantes, responsables des Myiases cavitaires et cutanées chez les animaux domestiques el sauvages d'Afrique equatoriale (Tehad, R. C. A., Congo) :

Rhinoestrus purpureus (BRAUER, 1858) : ânes.

Oestrus ovis (LINNÉ, 1761) : moutons et chèvres.

Oestrus variolosus (LOEW, 1863) : Damaliscus korrıgum (Ogilbb).

Gedeistia cristato: Alcelaphus lelwel.

Kirkioestrus blonchardi (GEDOELST, 1914) : Damalistus korrigum (Ogil).

Kirkiøstrus minutus: Alcelaphus lelwel (HEUGLIN).

Cephalopina titiliotor (CLARK, 1816) : dromadaires.

Crivellia corinnae (CRIVELLI, 1862) : Gazella dorcas (LINNÉ).

Gosterophilus nosolis (LINNÉ, 1758) : ânes et chevaux.

Gasterophilus intestinalis (DE GEER, 1776) : ânes et chevaux-chien.

Gosterophilus pecorum (FABRICIUS, 1794) : ânes.

Platycobboldia loxodontis (BRAUER, 1896) : Loxodonta africana (Blum.). Loxodonta africana cyclotis (MATSCHIE).

Neocuterebra squamosa (GRUNBERG, 1906) : Loxodonta africaba (Blum.).

Cordylobia anthropophago (E. BLANCHARD) : chien.
\end{abstract}

$2^{\circ}$ Le territaire de la République du Tchad est largement contamıné ainsi qu'en témoignent les cinq cartes jointes au présent document.

30 Le nombre d'animaux infestés est élevé :

Platycobboidia loxodontis : la totalité des éléphants autopsiés (7).

Crivellia corinnae : 12 des 26 gazelles dorcas autopsiées.

Esstrus variolosus : 4 des 11 damalisques autopsıées.

Gedolstio cristato: 1 des 14 bubales autopsiés.

Kirkıœstrus blanchardi : 1 des 11 damalisques autopsıées.

2 des 13 bubales autopsiés.

Kirkicestrus minutus: 1 des 14 bubales autopsiés.

Estrus ovis : de 43 à 55 p. 100 des 3.555 moutons autopsiés. de 15 à 18 p. 100 des 340 chèvres aulopsiées.

Cephalopina titillator : 29 des $\mathbf{4 5}$ dromadaires aulopsiés.

Rhinœstrus purpureus : 14 des 67 ânes autopsiés.

Gasterophitus intestinalis : 14 des 45 chevaux autopsiés.

50 des 67 ânes autopsiés.

Gasterophilus nasails : 36 des 67 ânes autopsiés.

14 des 45 chevaux autopsiés.

Gasterophilus pecorum : 6 des 67 ânes autopsıés.

$4^{\circ}$ Les auleurs donnent quelques renselgnemenis sur les variations saisonnières du parasitisme par CEstrus ovis chez le mouton.

Cette note est accompagnée de 67 références biblıographıques. 


\section{INTRODUCTION}

On désigne sous le nom de Myiases des affections lıées à la présence en différents points du corps de larves de Diptères appartenant surtout aux familles des Estridae, des Gasterophilidae et des Calliphoridae. Suivant leurs localisations, elles sont : cutanées, cavitaires ou intestınales. D'autres résultent d'infestation's de plaies préexistantes : ce sont les Myiases des plaies.

Chez les animaux domestıques, les agents des Myiases sont connus depuis fort longtemps. Malheureusement on ne sait toujours pas quel rôle pathogène exact il faut leur assigner, ce qui faisait écrire à DU TOIT et FIEDLER (1956), à propos d'Gistrus ovis :

«D'une manière générale le parasıte est considéré comme bénin ef responsable d'effets morbides relativement mınimes. D'un autre côté beaucoup de chercheurs estıment que l'irritation permanente produlte par les épines de cuticule et les pièces buccales des larves ajoutées à une certaine substance toxique excrétée par eux affectent profondément le bien-être des animaux infestés et que l'on doit considérer l'infestation sous un jour sérieux.»

De nombreux travaux, comportant souvent des inventaires détaillés, ont été publiés en divers points du globe. Ils intéressent :

- l'Europe (BRAUER, 1863, 1886, 1889, 1892 ef 1897 ; R. BLANCHARD, 1892 ; RAILLIET, 1908 ; PORTCHINSKY, 1906 et 1913 ; SURCOUF ef GUYON 1925 ; SÉGUY, 1937 ; NEVEU-LEMAIRE, 1938 ; BASHHAKOV, 1947 ; HENNIG, 1952 ; GRUNIN, 1955, 1957 ef 1961; SICCART, RUFFIE et MEIRA, 1958; ANTIPIN et Coll. 1956, MANSUY, 1959 ; DINULESCU, 1960 ; NOSIK ef GONCAROV, 1960, DAMIAN, 1961).

- I'Asie (STEEL, 1887 ; SONI, 1939 ; TSELICHTCHEVA et KRIVKO 1958).

- I'Australie (ROBERTS, 1940).

- I'Amérique (TOWNSEND, 1935 et 1938 ; COBBETT, $1940 a$ et $b$; FALLIS, $1940 ;$ COBBETT et MITCHELL, 1941; BABCOCK, 1953; BENNETT, 1955 ; SABROVSKY, 1957, MELENEY, COBBETT et PETERSON, 1962 ; MITCHELL et COBBETT, 1963).

Sur le continent africain, les recherches ont été menées le plus activement:
- En Afrique du Sud (SCHLEBEN, 1910. BEDFORD, 1925 et 1927 ; DU TOIT et CLARK, 1935 ; DU TOIT, 1935 ; DU TOIT et FIEDLER, 1956 ; ZUMPT, 1957 ; $1958 a$ et $b, 1959,1960$, $1961 a$ et $b, 1962 a, b$, et $c$; BASSON, $1962 a, b$ et $c)$.

- En Afrıque de l'Est (SJÖSTEDT, 1908 ; PILLERS ef EVANS, 1926 ; SYMES et ROBERTS, 1932; LEWIS, 1933; AUSTEN, 1934; LAURENCE, 1961).

- Au Soudan de l'Est (KING, 1911).

- Au congo ex-belge (R. BLANCHARD 1893 ef 1896; SCHOUTEN, 1912; GEDOELST, 1914, 1915, 1916, 1919 ef 1923, RHODAIN ef BEQUAERT, 1912, 1913, 1915, 1916, et 1919 ; RHODAIN, 1927).

- En Nigeria (UNSWORTH, 1948 et 1949).

- Au Sahara (SEGUY, 1933).

- En Afrique occidentale (BOUET et ROUBAUD, 1912; ROUBAUD, 1914 a et b; rapp. ann. Dakar, 1955, 1957 et 1958).

Au Tchad les agents des Myiases n'ont fait, jusqu'à présent l'objet d'aucune étude systématıque. On ne connaît pour cet immense territoire de $1.500 .000 \mathrm{~km}^{2}$, mêlant des clımats de type saharien à des climats soudano-guinéens, que les espèces suivantes : Kirkiœstrus blanchard, signalé par GEDOELST (1915) dans les sinus frontaux d'un bubale tué par le Dr DECORSE en bordure du Chari (mission Chari-Tchad, 1904). Platycobboldia loxodóntis dans l'estomac d'un éléphant abattu à Fort-Archambault (GEDOELST, 1915) et (Estrus ovis chez l'homme dans le massif de l'Emi Koussi (Mıré, Rıoux et Jarry, 1961).

Le but de la présente note est de donner un premier aperçu de la répartition des agents des Myiases au Tchad, du degré d'infestation des animaux domestiques ef sauvages des variations saisonnières du parasitisme par Esstrus ovis chez le mouton.

Pour ce fare, 3.856 moutons, 340 chèvres, 75 dromadaires, 45 chevaux, 67 ânes, 7 éléphants, 11 damalisqueș et 14 bubales on été autopslés de 1954 d̀ 1964 en divers points de la République du Tchad et de la R. C. A. et les larves de Diptères soigneusement récoltées. 
Les déterminations ont été effectuées à partir des larves du deuxième et du troisième stades seulement. Bien entendu, en la matière, il est recommandé (ZUMPT, 1961 a) de travailler de préférence sur les formes adultes. Outre qu'il n'est pas toujours facile d'obtenir la pupaison et l'éclosion des larves, la plupart des prélèvements ont été expédiés au Laborataire soit dans de l'eau formolée salée, soit dans l'alcool à $70^{\circ}$, ce qui empêche les élevages ultérieurs.

\section{I. - Les PARAsites eN CAUSE - LIEUX \\ DE RÉCOLTE - TAUX D'INFESTATION DES ANIMAUX DOMESTIQUES ET SAUVAGES}

La classification des Estridae S. Lat. (Diptera) a donné lieu à des discussions serrées pendant de nombreuses années. BRAUER ef BERGENSTAMM (1889) font entrer dans les (Fstridoe tous les Calyptrata pourvus d'un appareil buccal rudimentaire, le groupe étant à leur avis très homogène.

SEGUY (1928, 1937) place dans la famılle des Muscidae les sous-familles suivantes : Gasterophilinae, Gyrostigminae, Cobboldunae et Rutteniidoe et dans la famille des Tachinidae, les $\mathcal{E}$ strinae et les Hypoderminae. NEVEU-LEMAIRE (1938) décrit deux famılles : Estridae et Gostrophilidae avec les genres CFstrus, Rhincestrus, Cepholopsis, Pharyngobolus et Neocuterebra dans la première et les genres Gasterophilus et Cobboldia dans la seconde.

VAN EMDEN (1944) remanie la classification : Jes Gasterophilus sont placés dans les Acolyptrato, les Cephenomyia et les Cuterebra dans les Calliphoridoe, les Estres et les, Hypodermes dans la tribu des Phosinae (Tachinidoe).

HENNIG (1952 reprend les idées de BRAUER et BERGENSTAMM (1889). Les Ostridae sensu loto comprennent, selon lui, cing groupes:

10 Les Gasterophilus avec les genres Gasterophilus LEACH, Gyrostigmota BRAUER et Cobboldia BRAUER.

20 Les Cephenomyio avec le genre Cephenomyia LATREILLE et Phoryngomyia; SCHINER.

30 Les Fstrus avec les genres Pharyngobolus BRAUER, Neocuterebra GRÜNBERG, Kirkıoctrus RHODAIN et BEQUAERT, Rhincstrus BRAUER, Tracheomyla TOWNSEND, CEstrus LINNÉ,
Gedoelstio RHODAIN ef BEQUAERT et Cepholopino STRAND.

$4^{0}$ Les Hypoderma avec les genres Hypoderma LATREILLE, Edemogeno LATREILLE, Estromyio BRAUER et BERGENSTAMM, Dermocestrus BRAUER.

5o Les Cuterebro avec les genres Cuterebra CLARK, Rogenhofero BRAUER, Pseudobogeria BLAU, Allowattamyia TOWNSEND, Rogerio AUSTEN et Dermatobia BRAUER.

ZUMPT (1957) n'est pas absolument de cet avis en ce qui concerne les Gasterophilus et la position systématique du groupe Cuterebra. Finalement, il propose pour les Calyptrota, agents des Myiases, une classification que, pour plus de clarté, nous nous proposons de suivre au cours de cet exposé.

\section{A. - Famille des $\mathbb{C E s t r i d a e}^{-}$}

\section{fo Sous-famille des Fstrinae :}

a) Estrus ovis LINNÉ.

Hôtes habituels : Ovis aries. Copra hircus.

Hôte accidentel : Canis familiaris.

C'est une espèce cosmopolite largement répandue à la surface du contınent africain : Afrique du Nord. Afrique de l'Ouest (BOUET et ROUBAUD. 1912 ; ROUBAUD, 1914 a); MOREL 1955 57), au Congo ex-belge RHODAIN et BEQUAERT 1916 : RHODAIN, 1927), en Nigeria (UNSWORTH, 1948 ef 1949), au Soudan (KING, 1911), en Afrique orientale (SJÖSTEDT, 1908 ; SYMES et ROBERTS, 1932, LEWIS, 1933 ; en Afrique du sud (BEDFORD, 1925, 1927 ; DU TOIT, 1935 ; DU TOIT et CLARK, 1935 : DU TOIT ef FIEDLER 1956 ; ZUMPT, 1962 a).

Au Tchad, CEstrus ovis est un parasite très fréquent des cavités nasales et des sinus frontaux des moutons, ainsi qu'en témoıgnent le tableau ! ef la carte I qui concernent plus spécialement les zones sahéliennes.

Chez les chèvres les renseignements sont encore fragmentaires : de prime abord, les taux d'infestation paraissent moıns élevés:

Chez le chien, le parasitisme es̈t purement accidentel (un cas sur 90).

En général, les larves d'CEstres sont peu abondantes chez le mouton, (de 3 à 20 par animal). Classiquement, l'affection se traduit par 


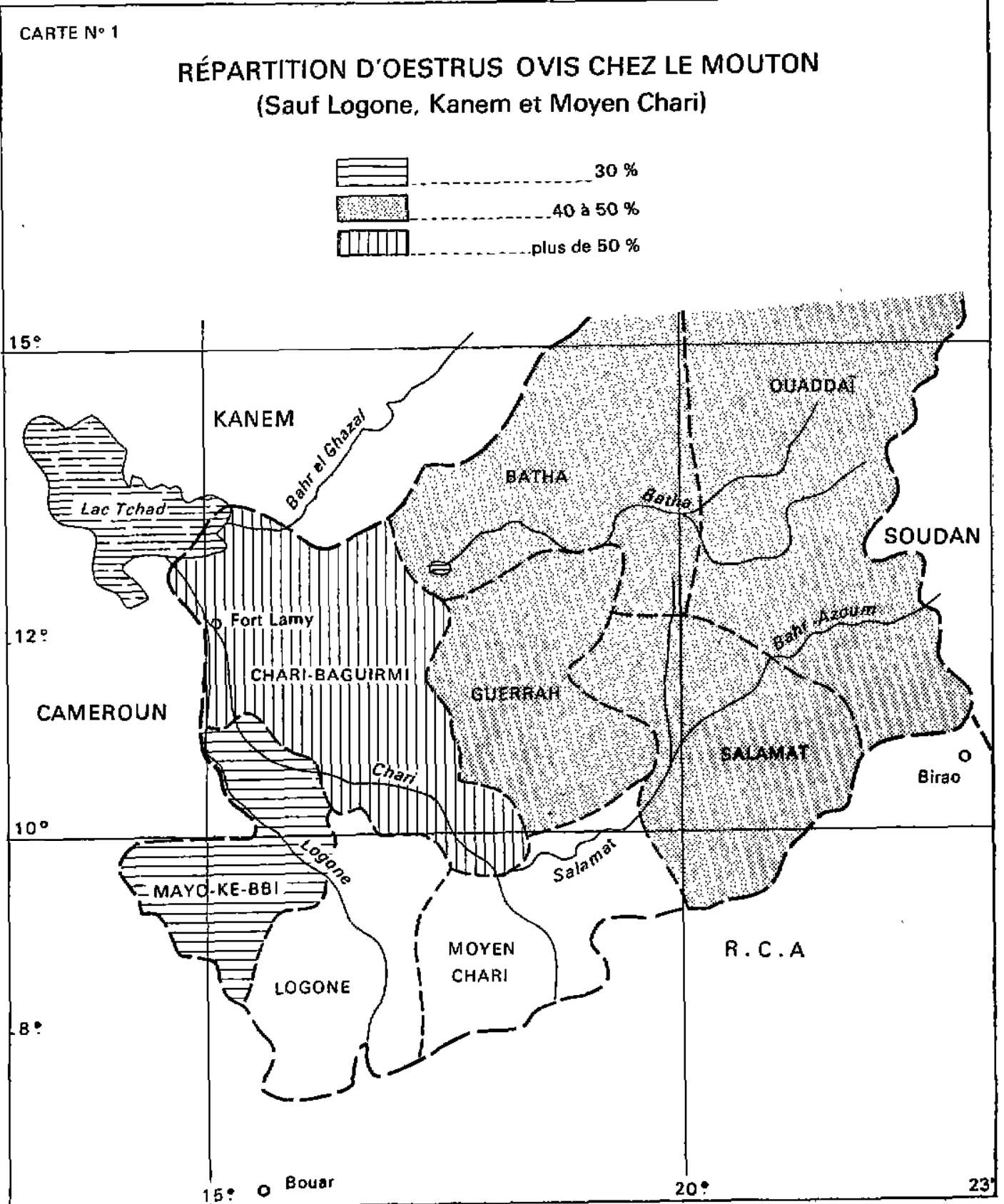


TABLEAU YO I

Óstrus ovis du mouton

\begin{tabular}{|c|c|c|c|}
\hline Régions & $\begin{array}{l}\text { Nombre de moutons } \\
\text { autopsiés }\end{array}$ & $\begin{array}{l}\text { Nambre de moutons } \\
\text { atternts }\end{array}$ & $\begin{array}{c}\text { Pourcentage d'anıamux } \\
\text { parasités }\end{array}$ \\
\hline $\begin{array}{l}\text { Chari-Bagunroi } \\
\text { (Ouest-Tohadien) } \\
\text { Batha } \\
\text { (Centre-Tchad) } \\
\text { Ourddai } \\
\text { (Est-Tchadien) } \\
\text { Mayo-Kebbi } \\
\text { (Sud-Ouest) } \\
\text { Total }\end{array}$ & $\begin{array}{r}1.996 \\
1.373 \\
379 \\
108 \\
3.856\end{array}$ & $\begin{array}{r}1.015 \\
672 \\
1666 \\
33 \\
1.886\end{array}$ & $\begin{array}{c}50,8 \mathrm{p} \cdot 100 \\
1 \\
49 \mathrm{p} \cdot 100 \\
\vdots \\
43,8 \mathrm{p} \cdot 100 \\
30 \mathrm{p} \cdot 100 \\
4 \\
48,8 \mathrm{p} \cdot 100\end{array}$ \\
\hline
\end{tabular}

TABLEAU NO II

Destrus ovis de la chèvre

\begin{tabular}{|l|c|c|c|}
\hline \multicolumn{1}{|c|}{ Régions } & $\begin{array}{c}\text { Nombre de chèvres } \\
\text { autopsiées }\end{array}$ & $\begin{array}{c}\text { Nombre de chèvres } \\
\text { parasitées }\end{array}$ & $\begin{array}{c}\text { Pourcentage } \\
\text { d'infegtation }\end{array}$ \\
\hline Chari-Baguirulit & 103 & 19 & $18,4 p \cdot 100$ \\
Ouaddai & 237 & 37 & $15,6 p \cdot 100$ \\
Total & 340 & 56 & $16,4 p \cdot 100$ \\
\hline
\end{tabular}

des « vertiges d'CEstres ou faux Tournis » qui, dans certaines parties du monde causent des dégâts considérables dans les troupequx. Au Tchad où l'élevage ovin et caprin est important ( 5 à 6 millions de têtes) I'Grstrose des sinus ne semble pas, jusqu'à plus ample informé, produire des troubles nı très sensibles, ni très apparents, sinon un jetage muco-purulent chronique surtout visible en hiver et au printemps. De temps en temps, l'animal secove la tête pour expulser les larves présentes dans les premières voies nasales. Les cas de «faux Tournis » semblent rares : en dix ans, nous n'avons pas eu l'occasion d'en observer. La mortalité paraît faible. Tous ces faits ne sont pas nouveaux et ROUBAUD (1914 a, p. 179) faisait déjà les remarques sui- vantes, valables pour le Sénégal : «Les moutons des races autochtones de l'Afrique occidentale ne semblent pas très sensibles au vertige d'F-stres. Nous les considérons, à ce point de vue, comme doués d'une véritable accoutumance naturelle, très intéressante et qui est du même type que celle qu'offrent les grandes antilopes sauvages à l'égard des nombreuses larves d'Fstres cavicoles qui les infestent. Les animaux ne paraıssent pas souffrır de la présence des parasites. Les éleveurs du Soudan ne les redoutent guère pour leurs troupeaux ; ils ne les considèrent que comme des hôtes gânants, très rarement susceptibles d'entraîner la mort. »

Selon les années et l'abondance des précipitations, le nombre des porteurs d'Fstres varie, 
TABLEAU No III

\begin{tabular}{|c|c|c|c|}
\hline Années & $\begin{array}{c}\text { Nombre de moutons } \\
\text { autopsiés }\end{array}$ & $\begin{array}{l}\text { Nombre de moutons } \\
\text { parasités }\end{array}$ & $\begin{array}{c}\text { Taur } \\
\text { d'infestation }\end{array}$ \\
\hline 1954 & 36 & 20 & 55 p.100 \\
\hline 1955 & 248 & 130 & $52 \quad$ p. 100 \\
\hline 1956 & 55 & 30 & $54.5 \mathrm{p} .100$ \\
\hline 1957 & 255 & 115 & $51 \quad$ p. 100 \\
\hline 1958 & 121 & 54 & p. 100 \\
\hline 1959 & 260 & 135 & $51 \quad$ p.100 \\
\hline 1960 & 299 & 93 & $31 \quad$ p. 100 \\
\hline 1961 & 113 & 40 & $35 \quad \mathrm{p} \cdot 100$ \\
\hline 1962 & 182 & 89 & $49 \quad \mathrm{p} \cdot 100$ \\
\hline 1963 & 238 & 169 & $72 \quad$ p. 100 \\
\hline 1964 & 218 & 140 & $64.2 \mathrm{p} .100$ \\
\hline (6 mois) & & & \\
\hline
\end{tabular}

au Tchad, dans des proportions appréciables, comme le démontre le tableau no III, qui n'intéresse que les régions ouest du Tchad :

Il est difficile de tirer des conclusions définitives de ces chiffres. Cependant, les années très sèches semblent les plus favorables d̀ l'éclosion de ce parasitisme.

\section{b) Estrus variolosus LOEW 1863.}

Hôtes : Damaliscus korrigum OGILBY.

Nombre d'anımaux infestés : 4 sur 11.

Lieux de récoltes (carte na II) : Massenyia, 195556, Bahr Azoum, 1955.

Estrus variolosus a été décrit pour la première fois par LOEW à partir d'exemplaires provenant des sinus frontaux d'un bubale africain. L'espèce a été retrouvée par la suite en de nombreux points de l'Afrique du Sud du Sahara : Etat d'Orange, province du Cap ; Soudan (KING 1911) ; Afrique occidentale (ROUBAUD, 1914 ; RHODAIN et BEQUAERT 1916); Afrique orientale (SJÖSTEDT 1908) et Afrique équatoriale (VAN EMDEN 1944, BEDFORD, 1927).

Estrus variolosus a été signalé chez Connochaetes tourinus BURCHELL, Alcelophus buselaphus PALLAS, Alcelophus lichtensteini PETERS, Damoliscus lunatus BURCHELL et Hippotrogus equinus DESMARET.

Parmi les nombreux Fstrinés mentionnés chez les grandes antilopes africaines, ZUMPT
(1961 a) n'en retıent finalement que trois : E Estrus variolosus LOEW, Fstrus bassoni ZUMPT, Gsstrus aureoargentatus RHODAIN ef BEQUAERT.

Les larves du troisième âge recueillies dans les sinus des damalisques du Tchad ont des caractères morphologiques qui les rapprochent étroitement d'Gstrus variolosus. Nous les laissons sous ce nom en attendant la confirmation par les insectes adultes.

c) Gedoelstia cristata RHODAIN et BEQUAERT 1913.

Hôte : Alcelaphus lelwel HEUGLIN.

Nombre d'animaux infestés : 1 sur 14.

Lieu de récolte (carte no 11): Aouk, 1964.

Découvert au Katanga chez Alcelaphus lichensteini PETERS, cet $\mathcal{E}_{\text {stre a }}$ a éé décrit pour la premıère fois en 1913 par RHODAIN et BEQUAERT. Cette espèce a été retrouvée par la suite jusqu'en Afrique accidentale (Mali) et en Côte-d'ivoire.

Au Tchad, sur 14 A, lelwel HEUGLIN autopsiés en 1964, un seul provenant de la région du BahrAouk (préfecture du Salamat), a été trouvé porteur de larves du 3 e âge de Gedoelstia cristata.

d) Kirkiæstrus blanchardı GEDOELST 1914.

Hôtes : Alcelophus lelwel HEUGLIN.

3 parasités sur 14 . 
Damaliscus korrigum OGILBY.

1 seul parasité sur 11 autopsiés.

Lieux de récoltes: Guerrah Salamat (carte II).

Les premières larves de $K$. bionchardı ont été recueillies sur A. lelwel en 1904 par le Dr DECORSE lors de la mission Cheri-Tchad. Depuis, d'autres récoltes ont été faites: dans la région
Sénégal-Niger (Dr. DRAMARD) sur A. maior BLYTH, en Côte-d'Ivare (Dr. BOUET), en Afrique orientale portugaise (M. S. A. NEAVE) sur A. Lichensterni PETERS, en Afrique centrale et tropicale (VAN EMDEN 1944). En 1958, le Dr. MOREL (Dakar) signale cette espèce sur Bubale de Luzarche à Niokolo-Koba et sur A. lelwel aux environs de Bamako, à Boré.

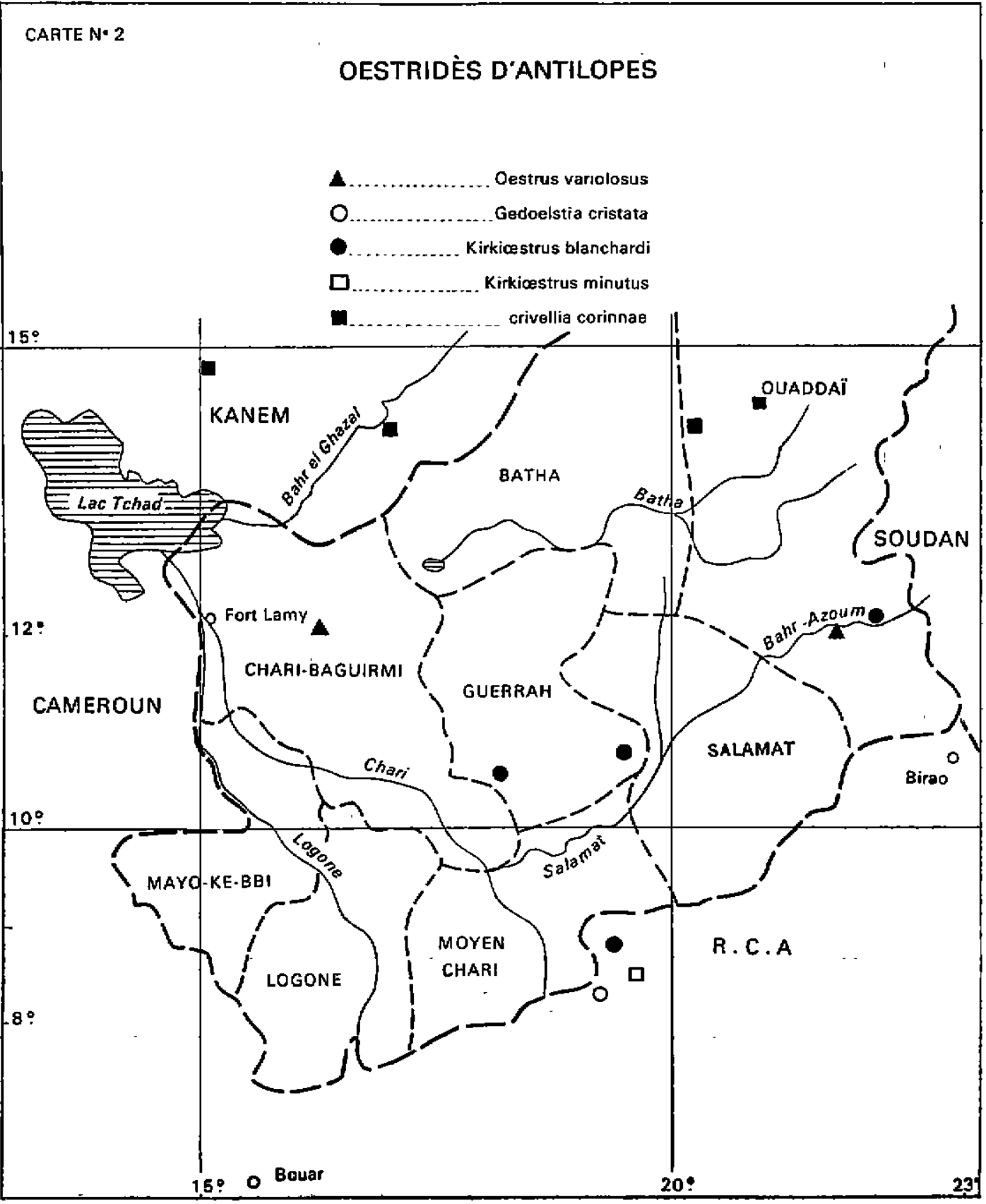


Récemment au Tchad l'autopsie de $14 \mathrm{~A}$. lelwel a révélé le parasitisme par K. blanchardi chez 3 d'entre eux. Ces Bubales provenaient des régions du Guerrah (environs de Mangalmé et de Melfi, 1957) et du Bahr Aouk (limite Tchad R. C. A., 1964).

Un seul D. Korrigum sur les 11 autopsiés provenant du Bahr-Azoum (préfecture du Salamał 1959) était porteur de 5 larves de K. blanchard au 3 e stade.

e) Kirkicestrus minutus RHODAIN et BEQUAERT 1916.

Hôte : Alcelaphus lelwel, HEUGLIN.

Nombre d'animaux infestés : 1 sur 14.

Lieux de récoltes : Bahr Aouk (limite Tchad R. C. A. 1961 carte II).

Signalé dans la plupart des régions africaines: Transvaal (BEDFORD, 1927) ; Congo, Kenya et Zululand (VAN EMDEN, 1944), RuandaUrundi (FAIN), cette espèce n'a été retrouvée au Tchad que sur un A. lelwel parmi les 14 autopsiés.

\section{f) Rhinœstrus purpureus BRAUER 1858.}

Hôte: Equus asinus L.

Nombre d'animaux infestés: 14 sur 67.

Lieux de récoltes: préfecture du Charı-Baguirmi, environs de Fort-Lamy (carte IV).

Cette espèce très cosmopolite affectant les Equidés sauvages et domestiques est sıgnalée en Afrique du Sud, au Tanganylka, au Soudan, qu Sénégal, en Afrique du Nord. Elle est rencontrée assez fréquemment au Tchad où les 14 ânes sur 67 autopsiés à Fort-Lamy étaient porteurs de larves au stade 3. g) Cephalopina titillator CLARK, 1816).

Hôte : Comelus dromedorius L.

Nombre d'animaux infestés : 54 sur 75 .

Lieux de récoltes: régions Nord du Tchad: (carte III).

fecture de Bıltine), - environs d'Arada (préfecture de Kanem), - environs de Nokou (préfecture du Ouddal),

- environs d'Abéché (pré-

La présence de cette espèce est liée à la distribution du genre Camelus ; elle se rencontre donc dans toute la zone sahélienne et désertique de I'Atrique septentrionale et a été également signalée dans le Sud-Ouest Africain (BEDFORD, 1927).

Au Tchad, les autopsies de 36 dromadaires de la région de Biltıne pratiquées en décembre 1954 ant donné qu total 314 larves, ce qui constitue une mayenne de 8 larves par animal. 275 larves, soit 87,6 p. 100 étaient du $3 e$ âge, les autres 12,4 p. 100 du 2e age.

Dans la région du Kanem, 4 dromadaires ont donné à l'autopsie, pratıquée en juin 1957, 141 larves soit une moyenne de 35 larves par animal. Ces larves comprenant des exemplaires au $3^{\mathbf{e}}$ stade (101, soit 72 p. 100$)$ et au $2^{\text {e }}$ stade (40, soit 28 p. 100).

Les 14 dromadarres autopsiés à Abéché en avril 1958 ont donné 216 larves soit une moyenne de 15 par ındividu ; 188 (87 p. 100) étant au stade 3 et le reste $(13 \mathrm{p} .100)$ au stade 2.

Le tableau IV résume ainsi les résultats :

Ces résultats semblent démontrer que le parasitisme est le plus intense à l'époque de l'année où la sécheresse est maximum.

TABLEAU NO IV

\begin{tabular}{|c|c|c|c|c|c|}
\hline Locelités & $\begin{array}{c}\text { moyenne } \\
\text { larves/and. }\end{array}$ & $\begin{array}{l}\text { larres au } \\
\text { stade III }\end{array}$ & $\begin{array}{l}\text { Larree au } \\
\text { stade II }\end{array}$ & époque & $\begin{array}{l}\text { pourcentage } \\
\text { parasités }\end{array}$ \\
\hline Arada & 8 & 87,6 p.100 & 12,4 p. 100 & décembro & 73,4 p.100 \\
\hline Abéché & 35 & $72 \quad$ p.100 & $28 \quad$ p. 100 & juin & $66.6 \mathrm{p} .100$ \\
\hline Nokou & 15 & 87 p.100 & p.100 & avril & p. 100 \\
\hline
\end{tabular}




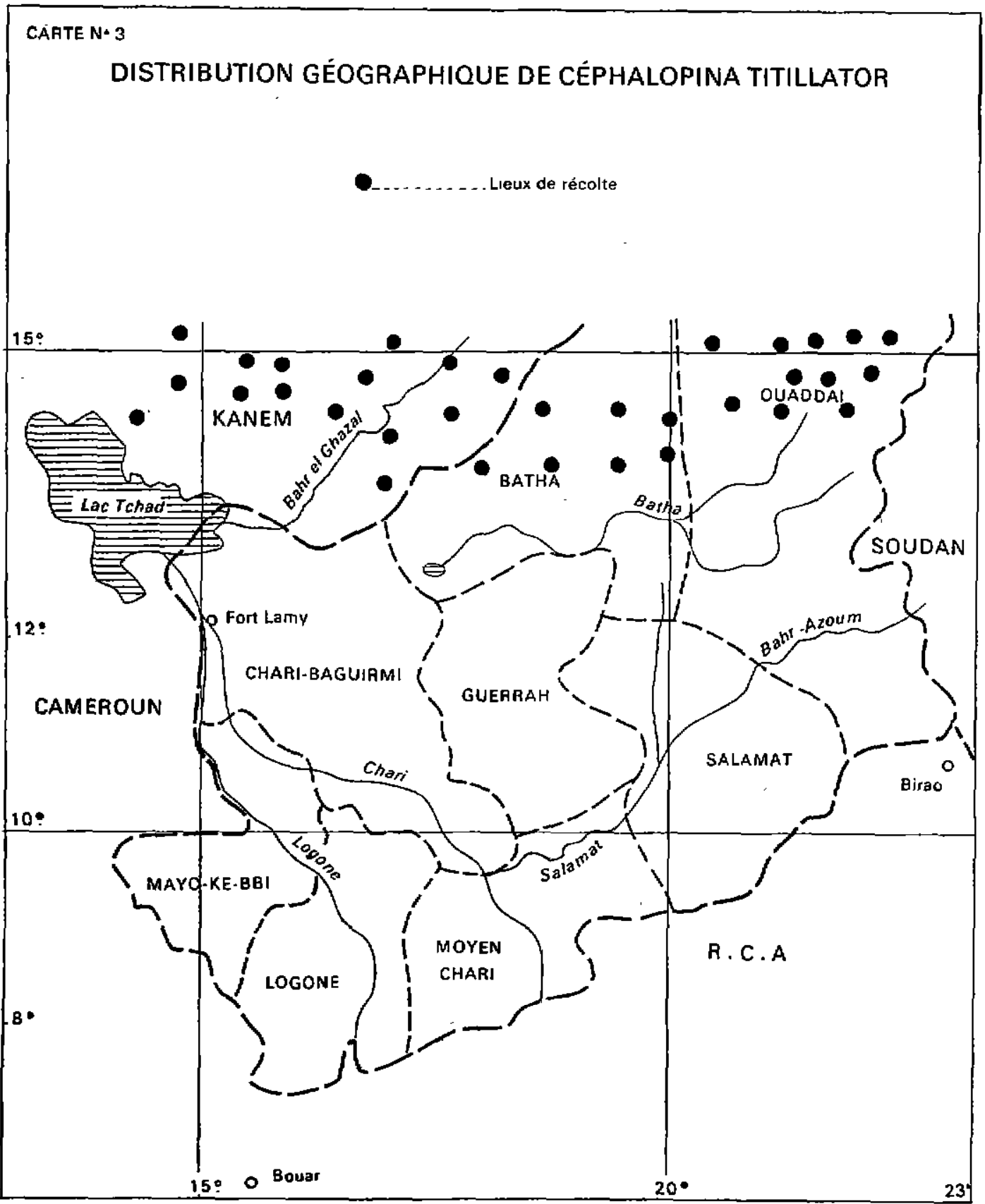




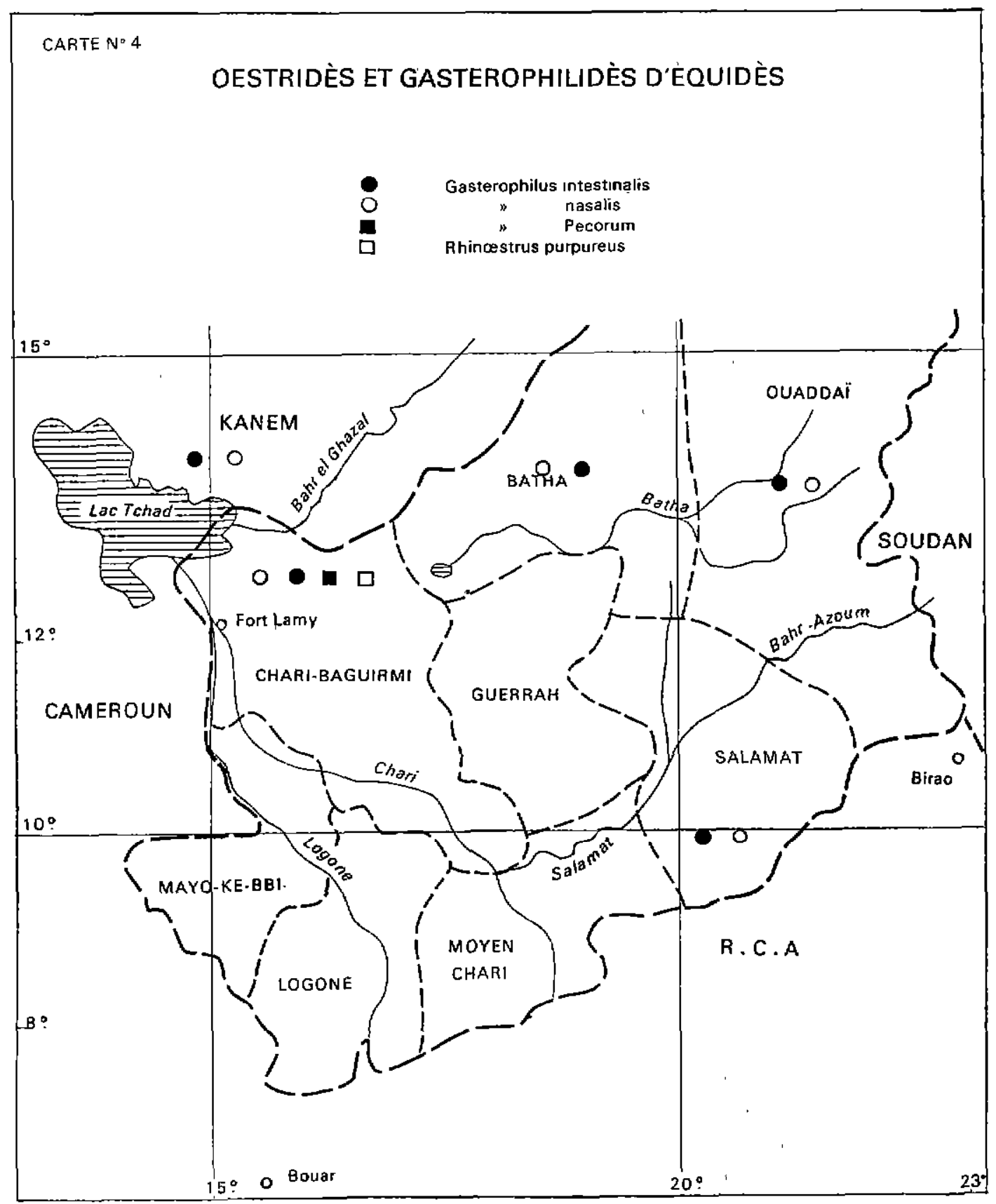


$2^{\circ}$ Sous-famille des Hypoderminae.

Crivellia corinnae CRIVELLI 1862.

Hôte : Gazella dorcos L.

Animaux infestés : 12 sur 26 autopsiés.

Origine : Arada (préfecture de Biltine) 1954: (carte II).

10 animaux sur 24.

- Moussoro (Bahr el Ghazal) 1958 :

1 animal sur 1

- Nokou (préfecture du Kanem)

1957

1 anımal sur 1.

En outre, quelques exemplaires ont été recueıllis sur le sol par la mission Berlıet (1960), au cours de l'exploration du Teneré (Sahara).

\section{B. - Famille des Gasterophilidae}

10 Sous-famille des Gasferophilinae.

a) Gasterophilus nasalis L. 1758.

Hôtes: Equus asınus L.

Equus cabollus L.

Nombre d'animaux parasités :

- Equus asinus : 36 sur 67.

- Equus cabollus : 14 sur 45.

Cette espèce très cosmopolite se rencontre dans toutes les régions où vivent des Equidés. Elle a d'abard été mise en évidence par le Dr JOYEUX (in ROUBAUD 1914a) à Kouroussa en Haute-Guinée et retrouvée ensuite dans toute l'Afrique.

Le tableau $V$ résume les résultats de 67 autopsies d'ânes et de 45 autopsies de chevaux pratiquées au Tchad:

\section{TLBLEAD NO V}

\begin{tabular}{|l|l|r|}
\hline Lieux de récolte & sur ânes & sur chevaux \\
\hline Chari-Bagucrmi & 34 sur 65 & 7 sur 27 \\
Batha & & 3 sur 6 \\
Ouaddai & 1 sur i & 1 sur 3 \\
Mojen-Charl & & 0 sur 5 \\
Kanem & 1 sur 1 & 3 sur 4 \\
Total & 36 sur 67 & 14 sur 45 \\
& & \\
\hline
\end{tabular}

(Carte no IV) b) Gosterophilus infestinalis DE GEER. 1776.

Hôtes: Equus asinus L.

Equus cabollus $L$.

Canis fomillaris $\mathrm{L}$.

Nombre d'animaux parasités :
E. asinus, 50 sur 67 .
E. coballus, 14 sur 45 .
C. familiaris, 2 cas.

Cette espèce également très cosmopolite se retrouve partoui où s'élèvent des Equidés. Elle apparaît comme la plus répandue des espèces de Gasterophiles Sur le contınent africain les premières récoltes viennent de l'Afrique occidentale : Sénégal-Nıger (BOUET), Guinée (Dr JOYEUX), du Soudan (ROUBAUD a), d'Egypte, du Cap (BRAUER), du Soudan angloégyptien ( $H$. KING).

Les résultats des enquêtes effectuées récemment au Tchad sont résumés dans le tableau $\mathrm{VI}$ :

TABLEAU NO VI

\begin{tabular}{|c|c|c|}
\hline Lieux de récolte & sur ânes & sur chevaux \\
\hline Char1-Baguirmi & 48 sur 65 & 8 sur 27 \\
\hline Batha & & 3 sur 6 \\
\hline Ouaddaí & 1 sur 1 & 1 siar 3 \\
\hline Dioyen-Chari & & 0 sur 5 \\
\hline Kanem & 1 sur 1 & 2 sur 4 \\
\hline TotaI & 36 sur 67 & 14 sur 45 \\
\hline
\end{tabular}

(Carte $n^{\circ} I V$ )

Gasterophilus infestinalis a déjà été trouvé accidentellement chez les Canıdés : chez l'hyène (BRAUER1863) et chez le chien (RAILLIET 1894). Récemment deux cas de ce parasitısme chez le chien viennent d'être signalés : I'un au MoyenCongo, l'autre au Tchad à Bongor (préfecture du Mayo-Kébı).

c) Gasterophilus pecorum FABRICIUS 1794.

Hôte: Equus asinus L.

Nombre d'anımaux infestés : 6 sur 67 . 
Lieux de récolte : les 6 ânes parasités proviennent d'animaux autopsiés à Fort-Lamy, originaires de la préfecture du Chari-Baguirmı. Ce parasite dont l'aire d'extension occupe toute la région éthiopienne n'a pas été retrouvé ailleurs au Tchad.

La fréquence du parasitisme par ces trols espèces de Gasterophiles rencontrées au Tchad se résume ainsı (Tabl. VII) :

\section{$2^{\circ}$ Sous-famille des Cobboldiinae.}

Platycobboldio loxodontis BRAUER 1896.

Hôte : Loxodonto africana BLUMEN BACH. Loxodonta ofricana cyclotes MATSCHIE.

Ceffe espêce se rencontre en Afrique noire, partout où vivent des pachydermes. Au Tchad, au Congo, au Cameroun et en R. C. A., elle a été observée sur la tatalité des sept animaux autopsiés.

\section{TABLEALU NO VII}

\begin{tabular}{|l|c|c|}
\hline \multicolumn{1}{|c|}{ parasitisme par : } & $\begin{array}{c}\text { Snes parasités } \\
\text { (sur } 67 \text { au total) }\end{array}$ & $\begin{array}{c}\text { chevaux parasités } \\
\text { (sur } 45 \text { au total) }\end{array}$ \\
\hline G. nasalis & 36 ânes $53,7 \mathrm{p} .100$ & 14 chevaux $31,1 \mathrm{p} .100$ \\
G. Intestinalis & 50 " $74,6 \mathrm{p} .100$ & 14 " $31,1 \mathrm{p} .100$ \\
G. pecorum & 6 " $8,9 \mathrm{p} .100$ & \\
\hline
\end{tabular}

(Carte $n^{0}$ IV)

Lieux de récoltes: (Carte no $\mathrm{V}$ ).

Fort-Foureau (Nord-Cameroun) 19541 éléphant Bongor (Mayo-Kebbi) 19573 -Salamat (Bahi-Azoum) 19551 Birco (R. C. A.) 19641 Congo : un animal mort au jardın zoologique de Brazzaville en 1955 (Dr ROUSSELOT).

Le nombre de larves recueillies est important 487 dans la région de Fort-Foureau, 260 au Salamat et 195 au Mayo-Kebbi.

$3^{\circ}$ Sous-famille des Neocuferebrinae.

Neocuterebra squamosa CRÜNBERG 190.

Hôte : Loxodonta africana BLUMENBACH.

Déjà signalé en Afrique centrale (Congo exBelge), ce parasıte de la sole pédieuse a été, retrouvé sur un éléphant abattu dans l'Est de la Républıque Centre Africaine (district de Birao).

\section{C. - Famille des Colliphoridae.}

\section{Cordylobio anthropophaga E. BLANCHARD.}

Les larves de cette espèce, répandue dans toutes les régions d'Afrique, déterminent une Myiase furonculeuse chez l'homme et divers mammifères domestiques (chıen, cheval, ) ou sauvages (rongeurs). Ce parasitısme a été étudié par ROUBAUD (1914), BLACKLOCK et THOMPSON (1923) ef CUTHBERTSON (1942).

Deux cas ont été récemment rencontrés au Tchad sur des chiens (Bongor ef Fort-Archambault) et un en R. C. A. toujours sur le même animal (Bouar).

\section{II. - VARIATIONS SAISONNIËRES DU PARASITISME PAR GSTRUS OVIS CHEZ LE MOUTON}

Selon les années la quantité de moutons parasités par Fstrus ovis L. (voir plus haut : Tableau III) varie dans des proportions appréciables ef il apparaît que les années sèches sont les plus favorables à l'installation de ce parasitisme.

Au cours d'une même année des varıations de taux d'infestation peuvent également être observées.

Le graphique ci-joint représente la varıation annuelle des moyen'nes mensuelles du nombre de parasites (larves au stade 2 et au stade 3 ). 


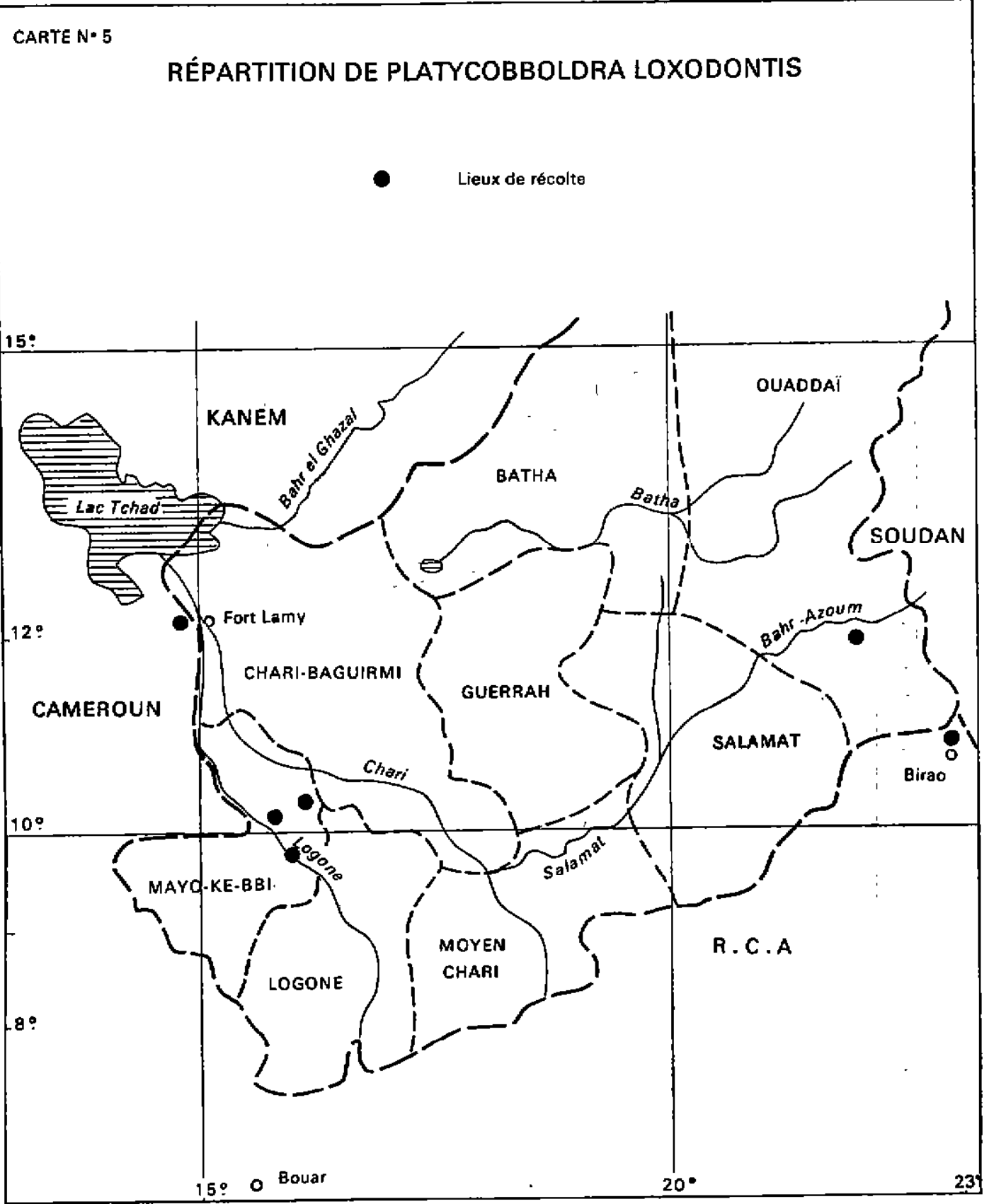




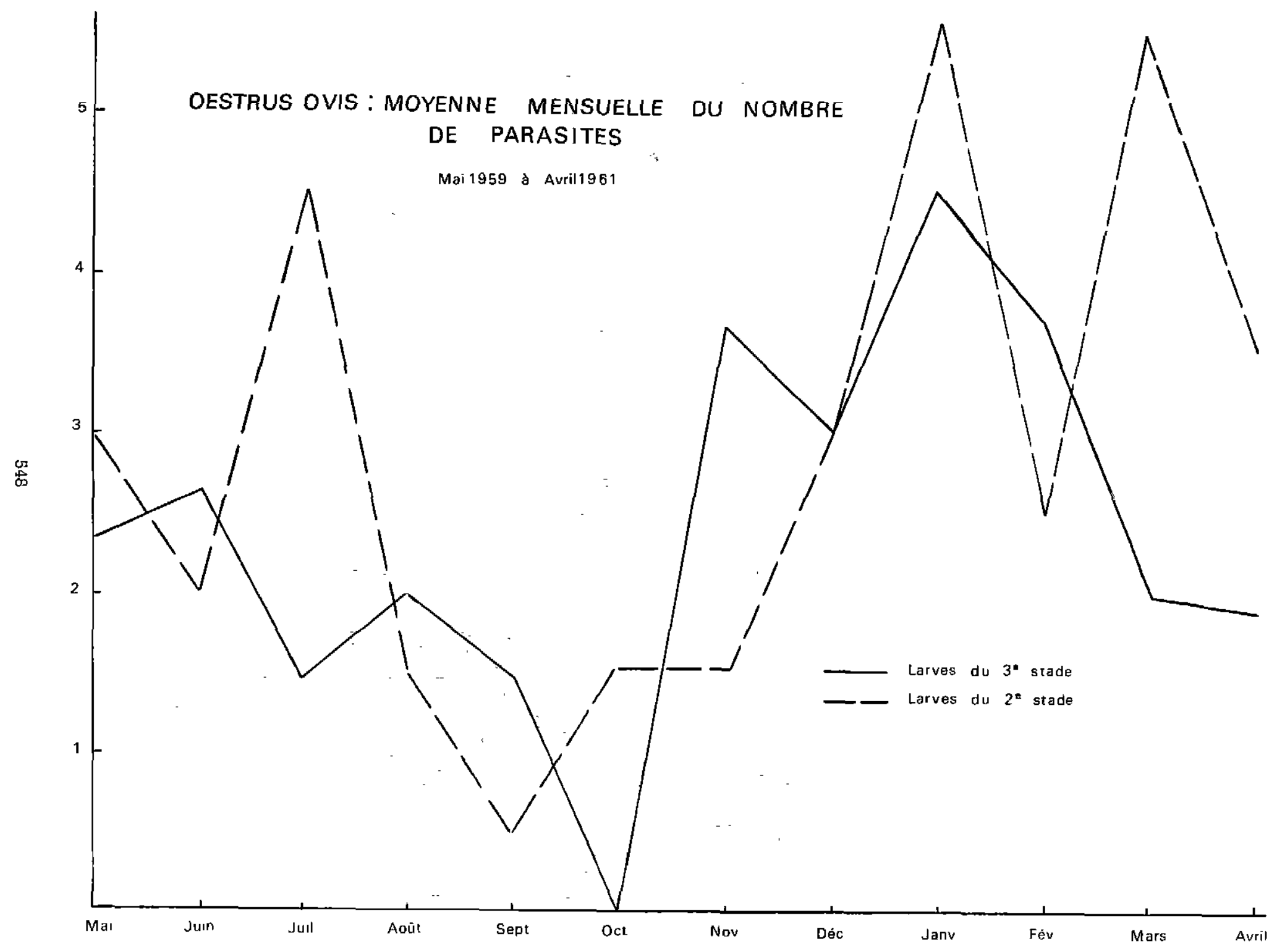


Ces moyennes ont été calculées sur une période de 2 ans (de mai 1959 à avril 1961) qui couvre une année où la saison des pluies avait été particulièrement précoce (1959) et une année normale (1960) où les premières pluies ne sont tombées que vers la mi-juın.

Gstrus ovis se retrouve chez le mouton à l'un ou l'autre stade de son évolution, toute l'année.

- Le maximum de larves au stade 3 (18 à $22 \mathrm{~mm}$ ), prêtes à être expulsées pour puper, se situe de novembre à mars. C'est seulement pendant cette pérıode qu'il a été possible d'obtenir l'éclosion des adultes à partir de larves mûres. Le taux d'infestation se maintient à un niveau assez bas jusqu'en septembre avec cependant une légère augmentation en juin. En octobre, fin de la saison des pluies, apparaît une chute complète de ce taux.

- Les larves du $2^{e}$ stade se rencontrent également toute l'année avec trois maxima en janvier. mars et juillet. En août, septembre et octobre (pleine saison des pluies); elles sont peu abondantes.

Les taux d'infestation paraissent done avoir un rapport direct avec les conditions climatiques. Ils sont maximum à la saison sèche et fraîche où les températures sont inférieures à $30^{\circ} \mathrm{C}$ et l'humidıté inférieure à 30 p. 100 . Ils diminuent progressivement pendant la. saison des pluies où la température est plus élevée et l'humidité toujours supérieure à 60 p. 100. Le taux minimum correspond aux périodes d'humıdité maximum. Ainsı, bien plus que l'élévation de tempérałure, l'augmentation de l'humidité est un facteur défavorable à l'installatıon du parasitisme par CEstrus ovis L.

Les transformations des larves du $2^{\mathbf{e}}$ stade en larves du $3^{e}$ ne s'effectuent pas en même temps et s'étalent sur une période assez longue au cours de l'année. De plus toutes les évolutions n'ont pas la même durée et au moins deux générations semblent être en présence. Ce phénomène déjà signalé au Nouveau-Mexique (U. S. A.) a également été observé par les Russes au Kazakhstan qui le nomment « dédoublement de la génération printanlère ».

\section{CONCLUSIONS}

$1^{\circ}$ Les auteurs notent l'existence des espèces suivantes, responsables de Myiases cavitaıres et cufanées chez les animaux domestiques ef sauvages d'Afrique équatoriale (Tchad, R. C. A., Congo, Cameroun).

Estrus ovis LINNÉ, 1761 : Ovis aries $(L)$, Capra hircus, Conis fomiliaris (LIN).

Estrus varilosus LOEW, 1863 : Damaliscus Korrigum (OGLILBY).

Gedoelstia cristato RHODAIN ef BEQUAERT 1913.

Alcelaphus leiwel (HEUGLIN).

Kirkicestrus bianchardi GEDOELST 1914 : Alcelaphus lelwel (HEUGLIN) ; Damoliscus Korrigum (OGLILBY).

Kirkicestrus minutus RHODAIN et BEQUAERT 1916: Alcelophus leiwel (HEUGLIN).

Rhincestrus purpureus BRAUER 1858 : Equus asınus (LINNÉ).

Cephalopina titillator CLARK 1816 : Camelus dromedarius (LINNÉ).

Crivellio corinnae CRIVALLI 1862: Gozella Dorcas (LINNÉ).

Gasterophilus nosalis LINNÉ, 1758 : Equus asinus (LINNÉ) ; Equus cabollus (LINNÉ).

Gasterophilus intestinalis DE GEER 1776 : Equus asmus (LINNÉ) ; Equus cabollus (LINNÉ) ef Canis familiaris (LINNE).

Gasterophilus pecorum Fabricius 1794 : Equus asinus (LINNÉ).

Platycobboldia loxondontis BRAUER 1896 : Loxodonta africana (BLUMENBACH) et Loxodonta ofricono cyclotis (MATSCHIE).

Neocuterebro squamoso (GRÜNBERG, 1906) : Loxonda ofricano (BLUMENBACH).

Cordylobia onthropophogo E. BLANCHARD : Canis farmiliaris (LINNÉ).

$2^{\circ}$ Le territoire de la République du Tchad est largement contamıné, ainsı qu'en témoıgnent les anq cartes accompagnant le texte.

30 Le nombre d'animaux infestés est élevé :

48,8 p. 100 des 3.856 moutons autopsiés

16,4 p. 100 des 340 chèvres autopsiées

4 p. 100 des 11 damalısques autopsiés

1 p: 100 des 14 bubales autopsiés 


\begin{tabular}{|c|c|c|c|}
\hline Kirkicestrus blanchardi & 1 & p. 100 des & 11 damalisques autopsiés \\
\hline & 3 & p. 100 des & 14 bubales autopsiés \\
\hline Kirkicestrus minutus & 1 & p. 100 des & 14 bubales autopsiés \\
\hline Rhinoestrus pupureus & 14 & p. 100 des & 67 ânes autopsiés \\
\hline Cephalopina titllator & 54 & p. 100 des & 75 dromadaires autopsiés \\
\hline Crivellia corinnoe ..... & 12 & p. 100 des & 26 gazelles dorcas qutopsiées \\
\hline Gasterophilus nasals ........... & 36 & p. 100 des & 67 anes autopsiés \\
\hline & 14 & p. 100 des & 45 chevaux autopsiés \\
\hline Gasterophilus intestmalis.. & 50 & p. 100 des & 67 ânes autopsiés \\
\hline & 14 & p. 100 des & 45 chevaux autopsiés \\
\hline & 2 & p. 100 des & 90 chiens autopsiés \\
\hline Gasterophilus pecorum. & 6 & p. 100 des & 67 ânes cutopsiés \\
\hline 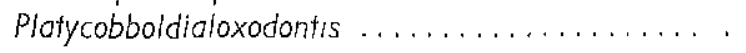 & 7 & p. 100 des & 7 éléphants autopsiés \\
\hline Necauterebra squamosa .................. & 1 & p. 100 des & 7 éléphants autopsiés \\
\hline Cordylobia onthropophoga.................. & 2 & p. 100 des & 90 chiens autopsiés \\
\hline
\end{tabular}

40 D'après les premiers sondages, les années sèches semblent être les plus favorables à l'éclosion de l'Estrose ovine. En cours d'année, le taux d'infestation est maximum en salson sèche : il diminue progressivement pendant la saison des pluies. L'augmentation du degré hygrométrique est un facteur défavorable à f'installation du parasitisme par $\mathrm{O}$. ovis.

\section{SUMMARY}

10 The authors have noted the existence of the following species, responsible for internal and external myiasis in wild and domestic animals of equatorial Africa (Chad, R. C. A., Congo).

Rhincestrus purpureus (BRAUER, 1858) : donkeys.

Estrus ovis (LINNÉ, 1761) : sheeps and goats.

Gedoelstio cristoto: Alcephalus lelwel.

Estrus variolosus (LOEW, 1863) : damaliscus korrigum (OGILBY). Kirkicestrus blanchardi (GODOELST, 1914): damaliscus korrigum (Ogil). Kirkiœstrus minutus : Alcelaplus lelwel (HEUGLIN).

Cepholopina tifiliator (CLARK, 1816) : dromedary. Crivelija corinnoe (CRIVELLI, 1862) : gazelia dorcas (LINNÉ).

Gasterophilus nasalis (LINNÉ, 1758) : horse and donkey.

Gasterophilus iniestinalis (DE GEER, 1776) : horse and donkay, dog. Gasterophilus pecorum (FABRICIUS, 1794) : donkey.

Platycobboldia loxadontis (BRAUER, 1896) : Loxodonta africana (Blum.). Loxodonta africana cyclotis (MATSCHIE).

Neocuterabro squamosa (GRUNBERG, 1906): Loxadonta africana. (Blum.).

Cordylobia anthropophaga (E. BLANCHARD) : dog.

$2^{0}$ The territory of the Republic of Chad is widely contamınated, as can be seen from the accompanying maps.

$3^{0}$ The number of animals infected is high :

Platycobboldia loxodontis : was found in all 7 elephants autopsied.

Crivellio corinnoe : 12 out of 26 gazelles dorcas autopsied.

Estrus variolosus : 4 out of 11 damaliscus autopsied.

Gedoelstia cristoto : 4 out of 11 hartebeestes outopsied.

Kirkiøestrus blanchardi : 1 out of 11 damaliscus autopsied.

2 out of 13 hartebeestes autopsied.

Kirkicestrus minutus : 4 out of 14 harlebeestes aulopsied.

Fstrus ovis : $43-55$ per, 100 of 3.555 sheeps autopsied.

$15-18$ per 100 of 340 goats autopsied. 
Cepholopina titlllator : 29 out of 45 dromedaries autopsied.

Rhinœstrus purpureus : 14 out of 67 donkeys autopsied.

Gasterophilus intestinalis : 14 out of 45 horses autopsied.

50 out of 67 donkeys autopsied.

Gasterophilus nasalis : 36 out of 67 don keys autopsied.

14 out of 45 harses culopsied.

Gasterophilus pecorum : 6 out of 67 donkeys outopsied.

40 The authors give some information on the seasonal variations of Fstrus ovis in the sheep.

This paper is accompanied by 67 references.

\section{RESUMEN}

Estudio de los agentes de las miasis en los animales domesticos y salvajes de Africa equatorial

10 Los autores señalan la existencia de las especies siguientes que ocasionan las miasis culá nea y cavitaria en los animales domésticos y salvajes de Africa equatorial (Tchad, R. C. A., Congo) :

Rhincestrus purpureus (BRAUER, 1858) : asnos.

Esstrus ovis (LINNÉ, 1761) : ovejas y cabras.

CEstrus variolosus (LOEW, 1863) : Damaliscus korrigum (Olgibb).

Gedolstio cristato: Alcelaphus lelwel.

Kirkicestrus blanchordı (GEDOELST, 1914) : Damaliscus korrigum (Ogil).

Alcelaphus lelwel (HEUGLIN).

Kirkicestrus minutus: Alcephalus lelwel.

Cephalopina titillotor (CLARK, 1816) : dromedarios.

Crivellio corinnae (CRIVELLI, 1862) : Gazella dorcas (LINNÉ).

Gasterophilus nasalis (LINNÉ, 1758) : asnos y caballos..

Gosterophilus intestinolis (DE GEER, 1776) : asnos y caballos, perro.

Gasterophilus pecorum (FABRICIUS, 1794) : asnos.

Platycobboldia loxodontis (BRAUER, 1896) : Loxodonta africana (Blum.). Loxodonta africana cyclotis (MATSCHIE).

Neocuterebra squomosa (GRÜNBERG, 1906) : Loxodonta africana (Blum.).

Cordylobio anthropophaga (E. BLANCHARD) : perro.

$2^{\circ}$ El territorio de la Republica del Tchad esta infectado ampliamente como lo muestran los cinco mapas adiuntos al presente documento.

30 El número de los animales infectados es elevado.

Platycobboldio loxodontis : En la totalidad de los 7 elefantes aulopsiados.

Crivellia corinnae : 12 entre las 26 gacelas dorcas autopsiadas.

CEstrus variolosus : 4 entre los 11 damaliscos ». Gedalstio cristata : 4 entre los 14 búbalós.

Gedalstia cristato: 4 entre los 14 búbalos.

Kirkiœstrus blanchardi : Kirkiœstrus minutus : 4 entre los 14 búbalos 1 entre los 11 damaliscos »2 entre los 13 búbalos ».

Kirkicestrus minutus: 4 entre los 14 búbalos.

Estrus ovis : de 43 a 55 por 100 de los 3555 ovejas » de 15 a 18 por 100 de las 340 cabras $\gg$.

Cephalopina titillator : 29 entre los 45 dromedarios».

Rhincestrus purpureus : 14 entre los 67 asnos ».

Gasterophilus intestinalis : 14 entre los 45 caballos ». 50 entre los 67 asnos 3 .

Gosterophilus nosolis : 36 entre los 67 asnos ». 14 entre los 45 caballos $»$.

Gasterophilus pecorum : 6 entre los 67 asnos».

40 Los autores dan algunos informes sobre las variociones según las estaciones del parasitismo causado por Fstrus ovis en la oveja.

67 referencias bibliograficas acompanan la presente nota. 


\section{BIBLIOGRAPHIE}

AUSTEN (E. E.) (1934). - Two new Oestridde (Diptera) parasitic in African antelopes. Ann. Mag. Nat. Hist., ser. 10, 14, 242-250.

BASSON (P. A.) (1962 a). - Sfudies on specific oculo-vascular myiasis of domestic animals : I. Historical Review. Onderstepoort J. Vet. Res., 29, I, 81-87.

BASSON (P. A.) (1962 b). - studies on specific oculo-vascular myiasis of domestic animals : II. Experimental transmission. Onderstepoort J. Vet. Res. ; 29, 2, 203-209.

BASSON (P. A.) (1962 c). - Studies on specific oculo-vascular myiasis of domestic animals : III. Symptomatology, pathology, aetiology and epizootiology. Onderstepoort J. Vet. Res. ; $29,2,211-240$.

BEDFORD (G. A. H.) (1925). - The sheeps nasalfly Oestrus ovis L. ; J. dep. Agr. S. Afr. ; II, 2., 119-123.

BEDFORD (G. A. H.) (1927), - Check list of the Muscidae and Oestridae which cause myiasis in man and animals in South Africa. $11^{\mathrm{e}}$ and $12^{\mathrm{e}}$ Reports Vet. Res. S. Afr., Part I; p. $483-491$.

BENOIT (P. L. G.) (1957). -De obligaat. Myiasis verwekkende; Liptera von Belgish Congo. Med Landbouwhogeschool Gent. ; 22, p. 654-669.

BLANCHARD (R.) (1856). - Sur un Oestre du Congo. Ann. Soc. Ent. Fronce.

BLANCHARD (R.) (1892). - Sur la présence de larves d'Oestrus ovis $L$. chez la chèvre. Bull. Sci. Fr. Belg. ; p. 256-258.

BLANCHARD (R.) (1893). - Contribution à l'étude des Diptères parasites; IV : sur une larve extraite du sinus frontal d'une antilope. Bull. Soc. Ent. France. p. 132-134.

BOUET (G.) et ROUBAUD (E.) (1912). — L'Oestre des moutons au Sénégal. Bull. soc. Path. Exot. ; $5,9,733-736$.

BRAUER (F.) und von BERGENSTAMM (1889). Die Zweifluger des Kaiserlichen Museums zu Wien IV Vorarbeiten zu einer Monographie der Muscaria Schizometope Pars 1 Denkschr k. Ak. Wiss wien math. naturwd. 56. p. 69-180.
DINULESCU (G.) (1960). - Observations sur la classification des Oestrides. Acad. R. P. R. Stud. Cer. Biol. (sect. Bio. Anim.) ; 12, $1 ; 7-20$.

DU TOIT (R.) et CLARK (R.) (1935 a). - The sheeps nasal-fly. A method of treatment for sheeps infested with the larvae of Oestrus ovis. J. S. Afr. Vet. Med. Assoc. ; 6, 1, 25-32.

DU TOIT (R.) (1935 b). - The sheep nasal worm. A method of treat. Farming in S. Afr. ; 10, 110, p. 197-198.

DU TOIT (R.) et FIEDLER (O. G. H.) (1956), Nlle methode de traitement de moutons infestés par O. ovis L. and J. V. R. 27, p. 6775.

GEDOELST (L.) (1914). - Note sur un genre nouveau d'Oestride. Bull. Soc. Path. Exot. ; 7 , p. $210-212$.

GEDOELST (L.) (1915). - - Note sur les Oestrides I. Rev. Zool. Afr. ; 4, p. 114-61.

GEDOELST (L.) (1916). - Note sur les Oestrides II. Rev. Zool. Afr. 4, 259-64.

GEDOELST (L.) (1923). - Les trois stades larvaires de Cobboldia loxodontis. Ann. Par. Hum. Comp. I ; p. 354-62.

HENNIG (W.) (1952). - Die larvenformen der Dipieren III. Akademieverlag, Berlin.

KARPENKS (S. E.) (1947), - Rhinoestrus of herses. Vetermanyia, 24, 42.

KING $\left(H_{i}\right)$ (1911), - Fourth Rep. Wellcome Res. Lab. Gordon M. Collège Khatoum, p. 127. LAURENCE (B. R.) (1961). - On a collection of Oestrid larvae (Diptera) from ear african game animals. Proc. Zool. Soc. London, 136, 4, 593-601.

LEWIS (E. A.) (1933), - Observations on some Diptera and Myiasis in Kenya Colony. Bull. Ent. Res. ; 24, 263-8.

MIRE (P.) de RIOUX (」. A.), JARRY (D.) (1961). - Oestres et œstroses sur le massif de l'Emi Koussi (Mission épidémiologique au Nord Tchad). Comité Coord. Sci. Sahara. p. 112114.

MOREL (P.). - cf. Rapports.

NEVEU-LEMAIRE (M.) (1938). - Traité d'Entomologie médicale et vétérinaire. Vigot, Paris, p. 860-912. 
PILLERS (A. W. N.) ef EVANS (A. M.) (1926). A new larva of Oestrus (Gasteroph) from zebras. Ann. Trop Med. Porasit. ; 20, p. 263266.

RAILLIET (A.) (1893). - Traité de Zoologie médicale et agricole. p. 762.

RHODAIN (J.) et BEQUAERT (J.) (1912), - Sur deux Oestrides nouveaux parasites du Potamochère et de l'Antilope chevaline au Congo Belge. Rev. Zool. Bot. Afr. ; 1, 3, 365-383.

RHODAIN (J.) et BEQUAERT (J.) (1913). - Gedoelstia cristoto nov. gen., nov. spe. Oestride parasite de Bubalis lichtensteini au Katanga. Rev, Zool. Afr, ; 2, p. 171-186.

RHODAIN (J.) et BEQUAERT (J.) (1915). - Sur quelques Oestrides du Congo. Sur quelques Auchmeromyies du Congo, Bull. Sac. Poth. Exot. ; 8, p. 452-461.

RHODAIN (J.) et BEQUAERT (J.) (1916). -Matériaux pour une étude monographique des Diptères parasites de l'Afrique. II. Revision des Oestrinae du Continent africain. Bull. Sci. Fr. Belg. ; 50, p. 53-165.

RHODAIN (J.) et BEQUAERT (J.) (1919). - Matériaux pour une étude monographique des Diptères parasites de l'Afrique: diptères de l'Eléphant et du Rhinocéros. Bull. Sci. Fr. Beig. ; 52, p. 379-465.

RHODAIN (J.) (1927). - Contribution à la faune des Oestrides du Congo Belge. Ann. Porasit. Hum. et Comparée. 5, p. 193-213.

ROUBAUD (E.) (1914a). - Etude sur la faune parasitaire de I'A. O. F. : part. I. Les producteurs de Myiases et agents similaires chez l'Homme et les animaux. Paris. Larose. 250 pages.

ROUBAUD (E.) (1914 b), - Oestrides gastricoles et cavicoles de l'Afrique occidentale française. Bull. Soc. Potho. Exot. ; 7, 3, 212-215.

SALEM (H. H.) (1935), - Myiasis in Egypt. J. Egypt. Med. Ass. : 18, p. 238-254.

SCHEBEN (L.) (1910). - Strobiloestrus oreotragi nov. sp., eine neve cestriden larven von Klippboch (Oreotragus sallatrix) und sonstige parasit ierende Dipteren aus Deustch. S. W. Africa. Zentralbl. Bakt. I. orig. 56, p. 50-4.

SCHOUTEN (H.) (1912), - Note sur l'hôte de l'Oestrus macdonaldi Gedœlst. Rev. Zool. East. Africa. ; 2, p. 142
SEGUY (E.) (1928). - Etude sur les mouches parasites, Oestrides ef Calliphorines d'Europe occidentale. Paris, 9, p. 1-12.5.

SEGUY (E.) (1933), - Mission saharienne Augieras. Draper 1927-28. Insectes Diptères. Bull. Muss. Hist. Not. ; 2, 5, p. 122-127.

SEGUY (E.) (1937). - Diptera, Fam. Muscidae, Gen. Insect. Vol. 205 ; 604 pages.

SICART (M.), RUFFIE (J.), MEIRA (M.) (1958). L'évolution larvaire de Oestrus ovis. Ann. Poras. Hum. et Comparée. 33, 3, p. 295.

SJOSTED (Y.) (1908). - Dipiera, Oestridae. Wiss. Ergebn. Schwed. Zool. exp. Kllimandjaro ; 2 , $10,11-24$.

SURCOUF (J.) et GUYON (L.) (1925). - Recherches préliminaires sur la morphologie ef la biologie des larves d'Oestres. Bull. Sóc. Ent. France. p. $66-72$.

SYMES (C. B.) et ROBERTS (J. I.) (1932). - A list of the Muscidae and Oestridae causing myiasis in man and animals in Kenya recorded at the Medical Research. Laboratory Nairobi. E. Afr. Med. J. : 9, p. 18-20.

UNSWORTH (K.) (19.48). - Observations of the occurence of Oestrus ovis in the nasal cavities and frontal sinuses of goats in Nigeria. Ann. Trop. Med. Parasit. ; 42, 2, 249-250.

UNSWORTH (K.) (1949). - Observations on the seasonal incidence of Oestrus ovis infection assum. Goats in Nigeria. Ann. Trop. Med. Parasit. ; 43, 3-4, 337-340.

VAN EMDEM (F. I.) (1944). - Keys to the Ethiopian Tachinidae. I. Phasiinae. Proc. Zool. soc. London. II4, part. 4, 398-436.

ZUMPT (F.) (1951). - Myiasis in man and animals in Africa. S. Afr. J. of Clinical Sci. : 2, 1, 38-69.

ZYMPT (F.) (1957). - Some remarks on the classification of the Oestridae s. lat. (Diptera), 20, 1, 154-161.

ZUMPT (F.) (1958). - On Rhinoestrus steyni, $n$. sp. and Gasterophilus zebrae R. et B. parasite of Burchell's Zebra. J. Ent. Soc. S. Afr. : 21-56.

ZUMPT (F.) (1958). - Remarks in the systematik position of myiasis producing flies (Diptera) of the african Elephant Lox. africana. Proc. R. Ent. Soc. London, 27, p. 8-14. 
ZUMPT (F.) (1959). - The Rhincestrus species of equids in Africa South of the Sahara (Diptera, Oestridae). Novo. Taxa. Ent. Mozambique ; 14, 10.

ZUMPT (F.) (1960), - The adult of Gasterophilus fernicictus Gedoelst and G. meridionalis Pillers ef Evans. J. Ent. Soc. S. Afr. : 23, 2, 411412.

ZUMPT (F.) (1961 a). - Oestrus bassoni nov. spec. ; a new nasal fly from South Africa. Novo. Taxa. Ent. Mozombique, 24, p. 1-13.

ZUMPT (F.) (1961 b). - The enigma of Strobiloestrus Braver (Dipt. Oestridae). Proc. R. Ent. Soc. London, 30, 7-8, 95-102.

ZUMPT (F.) (1962 c). - Oestrus bossoni nov. spec. ; ane nasal fly from South Africa (Dipt. Oestridae). Novos Taxa. Ent. 4, p. 1-3.

ZUMPT (F.) (1962 a). - The Oesireid flies of wild and domestic animals in the Ethiopian region, with a discussion of their medical and veterinary importance (Oestr. Gastero). Z. ang. Zool. 49, 3, 393-419.

ZUMPT (F.) (1962 b). - Die œstroiden liegen des Wildes in der æthiopischen region. Int. Kongr. Ent. WIEN. 2 (sect. 7), 454-457.

ZUMPT (F.) et BAURISTHENE (E.) (1962). - Tow new Rhincestrus from the Spring buck (Antidoreas Marsupalis) in south Africa. Noros Taxa. Ent. Mozambique, 28, p. 1-23.

Rapports annuels : I. E. M. V. P: T. Dakar 195557-58.

N. B. - La Bıbliographie citée ne concerne que les régions africaines au sud du Sahara. Pour plus de renseignements, consulter. le travail de ZUMPT (1962 a). 